\title{
Low-Frequency Finite-Element Modeling of the Gerbil Middle Ear
}

\author{
Nidal Elkhouri, ${ }^{1}$ Hengjin Liu, ${ }^{1}$ and W. Robert J. Funnell ${ }^{1,2}$ \\ ${ }^{1}$ Department of BioMedical Engineering, McGill University, 3775, rue University, Montréal, QC, Canada, H3A 2B4 \\ ${ }^{2}$ Department of Otolaryngology, McGill University, Montréal, QC, Canada
}

Received: 1 March 2006; Accepted: 31 July 2006; Online publication: 17 October 2006

\begin{abstract}
The gerbil is a popular species for experimental middle-ear research. The goal of this study is to develop a 3D finite-element model to quantify the mechanics of the gerbil middle ear at low frequencies (up to about $1 \mathrm{kHz}$ ). The $3 \mathrm{D}$ reconstruction is based on a magnetic resonance imaging dataset with a voxel size of about $45 \mu \mathrm{m}$, and an x-ray micro-CT dataset with a voxel size of about $5.5 \mu \mathrm{m}$, supplemented by histological images. The eardrum model is based on moiré shape measurements. Each individual structure in the model was assumed to be homogeneous with isotropic, linear, and elastic material properties derived from a priori estimates in the literature. The behavior of the finite-element model in response to a uniform acoustic pressure on the eardrum of $1 \mathrm{~Pa}$ is analyzed. Sensitivity tests are done to evaluate the significance of the various parameters in the finiteelement model. The Young's modulus and the thickness of the pars tensa have the most significant effect on the load transfer between the eardrum and the ossicles and, along with the Young's modulus of the pedicle and stapedial annular ligament, on the displacements of the stapes. Overall, the model demonstrates good agreement with low-frequency experimental data. For example, (1) the maximum footplate displacement is about $35 \mathrm{~nm}$; (2) the umbo/stapes displacement ratio is found to be about 3.5; (3) the motion of the stapes is predominantly
\end{abstract}

Correspondence to: W. Robert J. Funnell • Department of BioMedical Engineering - McGill University -3775 , rue University, Montréal, QC, Canada, H3A 2B4. Telephone: +1-514-398-6739; fax: +1-514398-7461; email: robert.funnell@mcgill.ca piston-like; and (4) the displacement pattern of the eardrum shows two points of maximum displacement, one in the posterior region and one in the anterior region. The effects of removing or stiffening the ligaments are comparable to those observed experimentally.

Keywords: finite element model, gerbil, low frequency, mechanics, middle ear, pedicle

\section{INTRODUCTION}

The middle ear is the site of many infections and birth defects, among other problems. Middle-ear prostheses and noninvasive diagnostic tools are often unsatisfactory. In attempts to improve our understanding of middle-ear mechanics, many groups have conducted experimental work on mammalian middle ears. In particular, gerbils are becoming popular for middle-ear research, in part because they are inexpensive and their middle-ear structures are easily accessible.

Mathematical models are useful for helping with the interpretation of experimental data and for providing additional insight. Lumped-parameter models were the first ones used to study the middle ear. Although these models are able to replicate some experimental data, their parameters are not closely tied to anatomical or physiological data. Nowadays, finite-element models are commonly used in middle-ear research. They are based on anatomical shapes, biological material properties, and realistic boundary conditions and loading conditions. The 
application of such models to middle-ear research was introduced by Funnell and Laszlo (1978).

A number of groups have since used the finiteelement method to study the mechanics of the eardrum and ossicles in humans (Wada et al. 1992; Beer et al. 1999; Bornitz et al. 1999; Prendergast et al. 1999; Koike et al. 2002; Sun et al. 2002) and in cats (Funnell and Laszlo 1978; Funnell et al. 1987; Ladak and Funnell 1996; Funnell et al. 2005). The geometries have generally been oversimplified and material properties have often been based on curve fitting. Although a number of experimental studies have been conducted on gerbil middle ears (von Unge et al. 1993; Rosowski et al. 1999; Olson and Cooper 2000; Dirckx and Decraemer 2001; Overstreet et al. 2003; Ravicz and Rosowski 2004), the only finiteelement model for the gerbil was a preliminary one presented by Funnell et al. $(1999,2000)$. The aim of this study is to present a detailed finite-element model of the gerbil middle ear and to quantify its mechanics at low frequencies based on a priori material-property estimates from the literature. No attempt was made to match experimental measurements by adjusting model parameters. The geometry was defined accurately with the use of magnetic resonance imaging and x-ray micro-CT data, supplemented by histological images, and of moiré topography. Sensitivity tests were performed to evaluate the significance of certain parameters in the finiteelement model. The behavior of the model was compared with previous experimental measurements on gerbil middle ears.

\section{FINITE-ELEMENT MODEL}

\section{Geometry and material properties}

As in our previous gerbil model (Funnell et al. 1999, 2000), the ossicles were generated from magnetic resonance microscopy (MRM) images with a voxel size of about $45 \mu \mathrm{m}$, and the eardrum was based on images from phase-shift moiré topography. Because of its extremely small thickness, the stapes annular ligament (SAL) in this new model was based on x-ray micro-CT images with a voxel size of about $5.5 \mu \mathrm{m}$. Histological serial sections were used to supplement the MRM and micro-CT images. For example, histology was very helpful in defining the exact points of origin and insertion of the ligaments and their shapes.

The model simulates the behavior of the gerbil middle ear in response to uniform sound pressures of low enough frequencies that inertial and damping effects may be neglected. At these frequencies, the middle-ear system is stiffness-dominated. Figure 1 shows the new model. For simplicity, each individual structure of the model was assumed to be homoge- neous with isotropic, linear, and elastic properties. Isotropy is used as an initial approximation because there is no strong direct evidence of anisotropy in the middle-ear structures. The structures of the model are the eardrum, the malleus, the incus, the stapes, the anterior mallear ligament (AML), the posterior incudal ligament (PIL), and the stapedial annular ligament (SAL). The mechanical properties of each structure are described in the following sections.

In the absence of experimental measurements, the Poisson's ratio was assumed to be 0.3 . Although 0.3 is a reasonable value for bone, it might be argued that Poisson's ratio should be close to 0.5 for soft tissues, because they are thought to be nearly incompressible. Values near 0.5 , however, cause numerical problems for the finite-element formulation used here. Increasing Poisson's ratio to 0.4 for the soft tissues in this model produced displacement changes of less than $10 \%$, consistent with previous modeling (e.g., Qi et al. 2004). This is also consistent with the theoretical relationship $G=E / 2(1+v)$ between Poisson's ratio $(v)$, Young's modulus $(E)$, and the shear modulus $(G)$, and the assumption that the annular ligament primarily operates in shear (Lynch et al. 1982). This relationship predicts that the effect of increasing Poisson's ratio to 0.5 would be only about $15 \%$ for the annular ligament. The effects for the eardrum, mallear ligament, and incudal ligament would be expected to be smaller because their configurations do not lead to major constraints on volume changes.

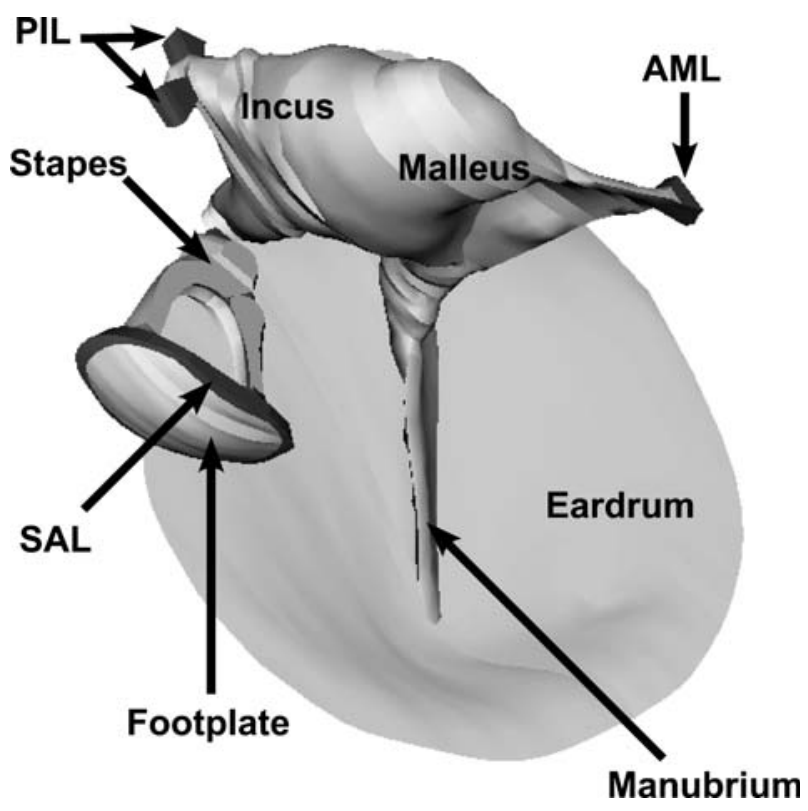

FIG. 1. Complete gerbil middle ear model. AML: anterior mallear ligament; PIL: posterior incudal ligament; SAL: stapes annular ligament. 
Eardrum. The eardrum is represented by 1101 triangular thin-shell elements. These elements include both bending and membrane (in-plane) stiffnesses. The geometry of the eardrum is based on phase-shift moiré topography, a noncontacting optical method that can measure the precise 3D shape of an object (Dirckx and Decraemer 1989, 1990). The Young's modulus of the pars tensa (PT) was set to $60 \mathrm{MPa}$, based on a recent study by Fay et al. (2005), who used theoretical methods to assess Young's modulus values for cat and human eardrums. For an isotropic cat model, Fay et al. (2005) estimated ranges for the Young's modulus of 30-60 MPa for the posterior region of the PT and $60-90 \mathrm{MPa}$ for the anterior region, so the value of $60 \mathrm{MPa}$ is consistent with our assumption of uniformity. The overall microstructure of the gerbil PT is similar to that of the human and cat $\mathrm{PT}$, and therefore $60 \mathrm{MPa}$ seems to be a reasonable estimate for the present model.

Given the lack of measurements on the mechanical properties of the pars flaccida (PF), Koike et al. (2002) assumed that the Young's modulus of the PF is one-third of that of the PT. Using the same ratio, we set the Young's modulus of the PF to $20 \mathrm{MPa}$. The $\mathrm{PT}$ and the PF were assigned uniform thicknesses of 7 and $24 \mu \mathrm{m}$, respectively, based on measurements by Kuypers et al. (2005).

The outer ends of the eardrum-where it meets the fibrocartilaginous ring-were fully clamped. In a finite-element model analysis of the mustached bat, Van Wijhe et al. (2000) simulated forces applied to the fibrocartilaginous ring and observed that the ring was much more rigid than the eardrum, at least at low frequencies.

Ossicles. To simplify the model and speed up the simulations, we modeled the ossicles as hollow shells composed of triangular surface elements, rather than as solid bodies filled with volume elements. For a given amount of surface detail, the representation as hollow shells greatly reduces the number of degrees of freedom in the finite-element solution by avoiding internal nodes. This is acceptable because the ossicles are assumed to behave like rigid structures, at least at low frequencies (Guinan and Peake 1967; Gundersen 1976), and because the mass distribution does not matter at frequencies low enough that inertial effects are negligible. The ossicles were represented with 4717 triangular shell elements.

The thicknesses of the shell elements for the ossicles were adapted to their geometries, based on direct measurements from the MRM images. In a slice that best showed the average shape of the solid structure, the approximate diameter was measured, and elements for that structure were given a thickness equal to about one-third of the diameter of the structure. In regions within a structure where the thickness changed considerably, different thicknesses were assigned. Thicknesses of 156,63 , and $10 \mu \mathrm{m}$ were assigned to the malleus head, neck, and manubrium, respectively. The thicknesses assigned to the incus were $156 \mu \mathrm{m}$ for the head, $50 \mu \mathrm{m}$ for the long process, $21 \mu \mathrm{m}$ for the lenticular plate, and $36 \mu \mathrm{m}$ for the posterior process. The thicknesses assigned to the stapes were $42 \mu \mathrm{m}$ for the head, $21 \mu \mathrm{m}$ for both crura, and $31 \mu \mathrm{m}$ for the footplate.

To ensure that the ossicles would behave rigidly, we tested a range of values for their Young's modulus. A final value of $80 \mathrm{GPa}$ was obtained, beyond which the displacements throughout the model would change by less than 3\%. By comparison, in recent finite-element models, the Young's modulus for human ossicles was taken to be 14.1 GPa (Prendergast et al. 1999; Sun et al. 2002) based on Speirs et al. (1999), who measured the Young's modulus of human ossicle allografts using axial compression; and $12 \mathrm{GPa}$ (Koike et al. 2002) based on a review by Evans (1973).

The pedicle of the incus was the only part of the ossicles to be represented with volume elements. This decision was based on the work done by Funnell et al. (2005), who concluded that the pedicle may not be rigid and may play a major role in the motion between the incus and the stapes. As in their model, a Young's modulus of $5 \mathrm{GPa}$ was assigned to the pedicle based on the measurements of Mente and Lewis (1994). The pedicle had an average thickness of $30 \mu \mathrm{m}$ and overall dimensions of $70 \times 140 \mu \mathrm{m}$.

The ossicles are linked together by two synovial joints: the incudomallear joint and the incudostapedial joint. The incudomallear joint was not modeled in this study because it has been observed to be rigid at low frequencies (e.g., Guinan and Peake 1967; Gundersen 1976). With respect to the incudostapedial joint, Guinan and Peake (1967) and Decraemer and Khanna (2000) reported relative motion between the incus and stapes, but Funnell et al. (2005) concluded that this may be attributable mostly to the pedicle, rather than to the incudostapedial joint - a conclusion that was confirmed by Robles et al. (2006) in the chinchilla. The incudostapedial joint was therefore not modeled in this study.

In the gerbil, the manubrium of the malleus is tightly connected to the eardrum along its whole length (Oaks 1967). Therefore, the attachment of the eardrum to the manubrium is represented as a direct connection of the two parts of the model.

Ligaments and muscles. According to Rosowski et al. (1999), a bony connection exists between the temporal bone of the middle ear and the anterior process of the malleus in gerbils. The histological section in Figure 2, however, appears to show connective tissue between the temporal bone and the malleus. Figure 3 presents a section from our MRM data depicting the 


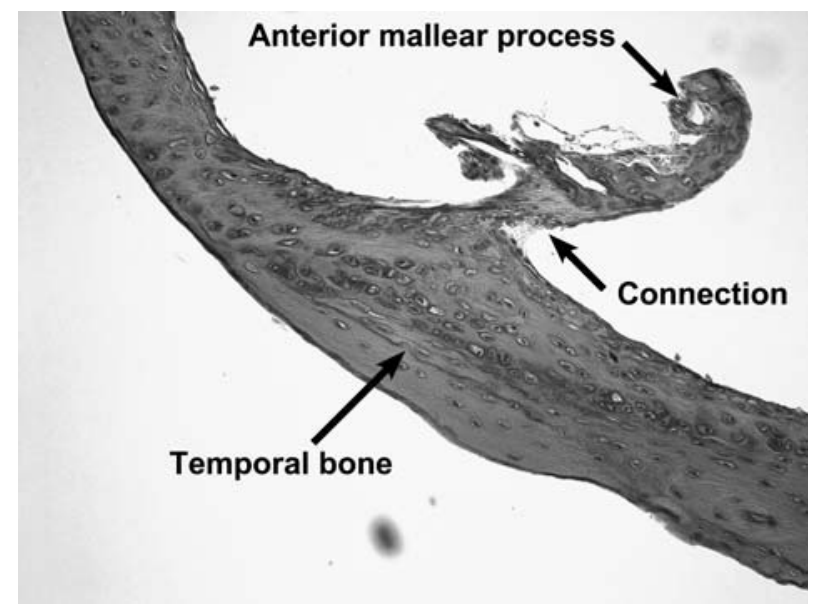

FIG. 2. Histological section of the connection between the temporal bone and the anterior process of the malleus, from a set of serial sections. There appears to be connective tissue between the temporal bone and the malleus, corresponding to the anterior mallear ligament.

same region in a different gerbil. The connection between the anterior process of the malleus and the temporal bone is a darker gray than the bone, which may indicate soft tissue. Based on these two images, the connection between the anterior process of the malleus and the temporal bone is modeled as a ligament.

The AML, the PIL, and the SAL were represented with 288, 457, and 2549 volume elements, respectively. To date, no experimental measurements of the elastic properties of the middle-ear ligaments have been done. Other research groups have fitted their models to experimental data to determine the Young's modulus of the ligaments (Prendergast et al. 1999; Koike et al. 2002; Sun et al. 2002). In this study, the Young's moduli of the AML and of the PIL were set to $20 \mathrm{MPa}$ as a reasonable value for dense connective tissue, as used in previous models from our laboratory (Ghosh and Funnell 1995; Funnell and Decraemer 1996; Ladak and Funnell 1996; Funnell et al. 1999, 2000). The AML is connected at one end to the anterior process of the malleus, and is clamped at the other end to represent its attachment to the cavity wall. The AML is about $180 \mu \mathrm{m}$ in height and about 140 $\mu \mathrm{m}$ in length, and its width varies between about 170 and $230 \mu \mathrm{m}$. The PIL is composed of two distinct bundles: one medial and the other lateral to the posterior process of the incus. Each bundle is connected to the incus at one end, and is clamped at the other end to represent its attachment to the cavity wall. Each bundle has a somewhat trapezoidal shape. The medial bundle is about $150 \mu \mathrm{m}$ in height and $100 \mu \mathrm{m}$ in length, and its width varies between about 70 and $160 \mu \mathrm{m}$. The lateral bundle is about $160 \mu \mathrm{m}$ in height and $50 \mu \mathrm{m}$ in length, and its width varies between about 130 and $200 \mu \mathrm{m}$. The Young's modulus of the SAL was set to $10 \mathrm{kPa}$, in line with vestibular sound pressure measurements by Lynch et al. (1982). This value was also used by Sun et al. (2002). The SAL follows the somewhat oval shape of the stapes footplate, with a large diameter of about $1 \mathrm{~mm}$ and a short diameter of about $750 \mu \mathrm{m}$. The ligament height varies between 50 and $170 \mu \mathrm{m}$ and its thickness varies between 10 and $20 \mu \mathrm{m}$.

The animal and human experiments that we used for comparison with our model were done either under anesthesia or postmortem. Under such conditions, the muscles are in a relaxed state. The passive mechanical effects of the two middle-ear muscles (tensor tympani and stapedius) are negligible: Dirckx and Decraemer (2001) found that at very low frequencies the passive muscles do not play a significant role in controlling ossicular motion. The middle-ear muscles were therefore omitted from this model. (This omission might have a significant influence on the simulations presented below in the section on the ossicular lever ratio with the stiffnesses of the AML and/or PIL set to zero.)

\section{Mesh resolution convergence}

Finite-element results depend in part on how fine a mesh is used to represent the geometry. The mesh resolution is defined in our software by an $x y$ resolution (within slices) and a $z$ resolution (the spacing between slices). As the mesh resolution increases, the computed displacements converge toward the exact solution for the model. However, the number of degrees of freedom also increases and strongly affects computation time. To determine an adequate mesh resolution for our model, we performed a series of runs by varying $x y$ and $z$ resolutions for every structure in the model, with smaller elements used where fine geometrical details need to be modeled. A mesh reso-

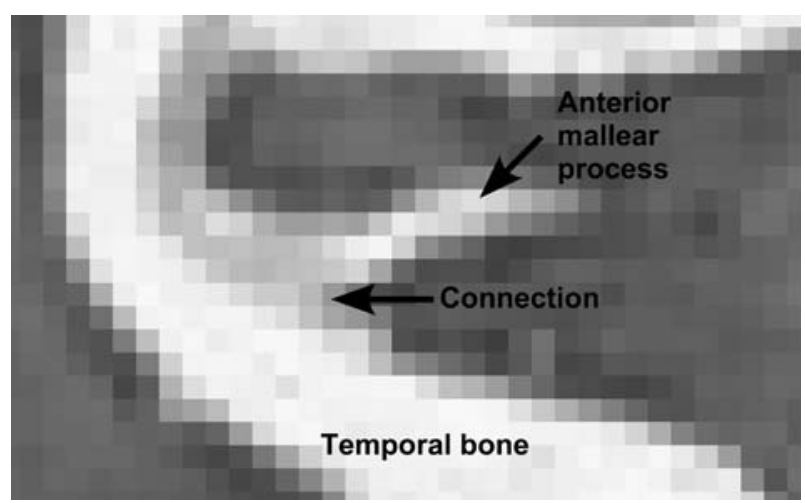

FIG. 3. MRM image of the connection between the temporal bone and the anterior process of the malleus. The grey value of the connecting part seems to be different from that of the surrounding bony structures, suggesting the presence of the anterior mallear ligament. Each pixel is $45 \mu \mathrm{m}$ square. 


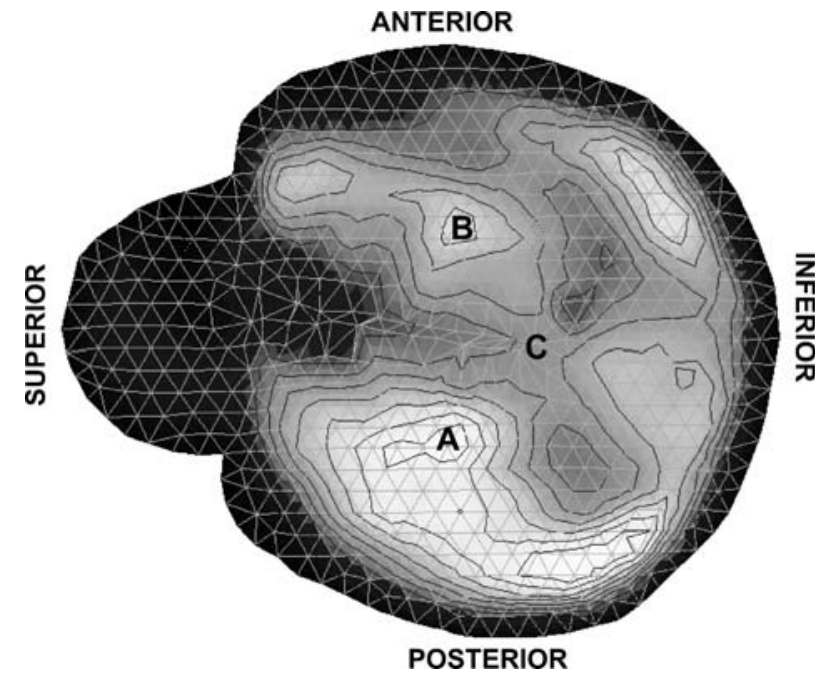

FIG. 4. Displacement pattern of the eardrum. (A) Point of maximum displacement in the posterior region of the eardrum. (B) Point of maximum displacement in the anterior region of the eardrum. (C) Umbo.

lution corresponding to a total of 9208 elements was chosen for the complete final model, the computation time for each run being less than $13 \mathrm{~min}$. Beyond that point, the computation time more than doubles but the displacements change by less than $3 \%$.

\section{BASIC MODEL RESULTS}

Simulation results for the complete gerbil finiteelement model-subjected to a uniform low-fre- quency sound pressure of $1 \mathrm{~Pa}$ applied to the eardrum-are presented in this section. Unless otherwise mentioned, the displacement values given are the magnitudes of the corresponding displacement vectors.

Figure 4 depicts the displacement pattern of the eardrum. A gray scale is used to represent the displacements, with black for zero displacements and white for the maximum displacement. Lines of constant displacement are also shown for clarity. In this case, the displacement values correspond to the vector components perpendicular to the plane of the tympanic ring (i.e., the plane of the figure). The maximum PF displacement is about $65 \mathrm{~nm}$. There are two points of maximum displacement on the PT: one in the posterior region (A) and one in the anterior region (B). The larger of the two maximum displacements is about $250 \mathrm{~nm}$ and lies in the posterior region. The displacement at the umbo $(\mathrm{C})$ is about $105 \mathrm{~nm}$.

Figure 5 shows the displacement vectors of the ossicles, as viewed along the apparent axis of rotation from the anterior and posterior views. The directions of the vectors clearly show the nature of the rotation about the axis, extending from the PIL to near the AML.

The footplate displacements are almost completely uniform. The difference between the maximum and minimum footplate displacements is only about $0.24 \%$. This implies a piston-like motion of the stapes, as shown in Figure 5. The transition from the rotational motion of the incus to the translational motion of the stapes apparently occurs in the flexible pedicle.
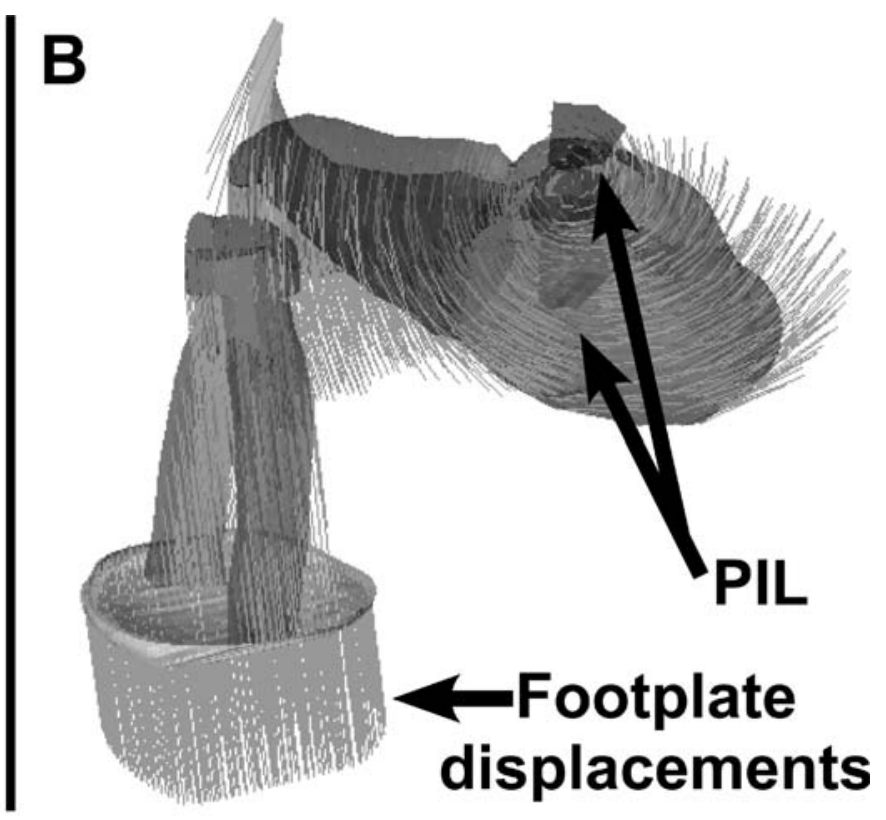

FIG. 5. (A) Anterior and (B) posterior views of the ossicles (eardrum and manubrium omitted for clarity). The displacement vectors clearly show the apparent axis of rotation through $(\mathrm{A})$ the anterior mallear ligament and $(\mathrm{B})$ the posterior incudal ligament. 


\section{SENSITIVITY ANALYSIS}

Sensitivity tests attempt to distinguish between the highly influential parameters, whose values must be carefully chosen, and those whose values have little effect on the low-frequency behavior of the model. The parameters investigated here were (1) the thicknesses of the pars flaccida and pars tensa ( $T_{p f}$ and $T_{p t}$, respectively) and (2) the Young's moduli of the pars flaccida, pars tensa, stapedial annular ligament, anterior mallear ligament, posterior incudal ligament, and pedicle $\left(Y_{p f}, Y_{p t}, Y_{s a l}, Y_{a m l}, Y_{p i l}\right.$, and $Y_{p e d}$, respectively). Effects on the footplate displacements will be examined first, followed by effects on the eardrum and umbo displacements. These displacements are measures of middle-ear output (footplate) and input (eardrum and umbo), and are commonly studied experimentally. The effects on the umbo/stapes ratio and the eardrum/umbo ratio will then be presented; these two ratios may be taken as measures of the ossicular lever ratio and of the load transfer between the eardrum and the manubrium, respectively. The effects of the AML and of the PIL on the axis of rotation of the ossicles will be examined. Finally, the effects of the shape of the eardrum on the overall behavior of the model will be presented.

\section{Footplate displacements}

The sensitivity of the model to its eight parameters is shown in Figure 6. Values for each parameter range from 0.1 to 3 times its nominal value. All curves are monotonic over this range. Overall, as expected, the displacements become smaller as the model becomes stiffer.

Excluding $Y_{a m l}$, when the parameters are decreased by a factor of 10 , the footplate displacements increase by $13 \%\left(Y_{p i l}\right)$ to $42 \%\left(Y_{p e d}\right)$. When the same parameters are increased by a factor of 3 , footplate displacements decrease by $13 \%\left(Y_{p i l}\right)$ to $56 \%\left(T_{p t}\right)$. For the $Y_{a m l}$ parameter, footplate displacements increase by only $0.6 \%$ at a ratio of 0.1 , and decrease by only $1.7 \%$ at a ratio of 3 .

The stapes annular ligament $\left(Y_{\text {sal }}\right)$ has a greater influence on footplate displacements than the anterior mallear ligament $\left(Y_{a m l}\right)$ and the posterior incudal ligament $\left(Y_{p i l}\right)$. A question arises as to whether $Y_{a m l}$ and $Y_{p i l}$ may affect the axis of rotation without much affecting the footplate displacement. This will be discussed below.

In a 3D finite-element model of a cat middle ear, Ghosh and Funnell (1995) represented the ossicles with a fixed axis of rotation and with a simple flexible connection between the incus and the stapes, providing a preliminary implementation of joint flexibility. They varied the incudostapedial joint stiffness from very small to very large values, and computed footplate displacements. For very low stiffnesses, the footplate displacement decreased because a very soft incudostapedial connection does not transmit force effectively. As the incudostapedial stiffness increased, footplate displacement reached a peak then dropped slightly and stabilized at a constant value.

To look for a similar phenomenon, we varied the stiffness of the pedicle $\left(Y_{p e d}\right)$ to extreme values in our model. The results are consistent with those of Ghosh and Funnell (1995). The stapes-displacement peak is at $100 \mathrm{MPa}$ in the present model. As the stiffness decreases below $100 \mathrm{MPa}$, the footplate displacements approach zero because the pedicle becomes too soft to transmit forces well. As the stiffness increases above $100 \mathrm{MPa}$ the footplate displacements again decrease, because the pedicle becomes too stiff to permit the transition from rotational motion to translational motion. The displacements reach a plateau at extremely high stiffnesses because the pedicle is so rigid that any further increase would have a negligible influence on footplate displacements. The peak here

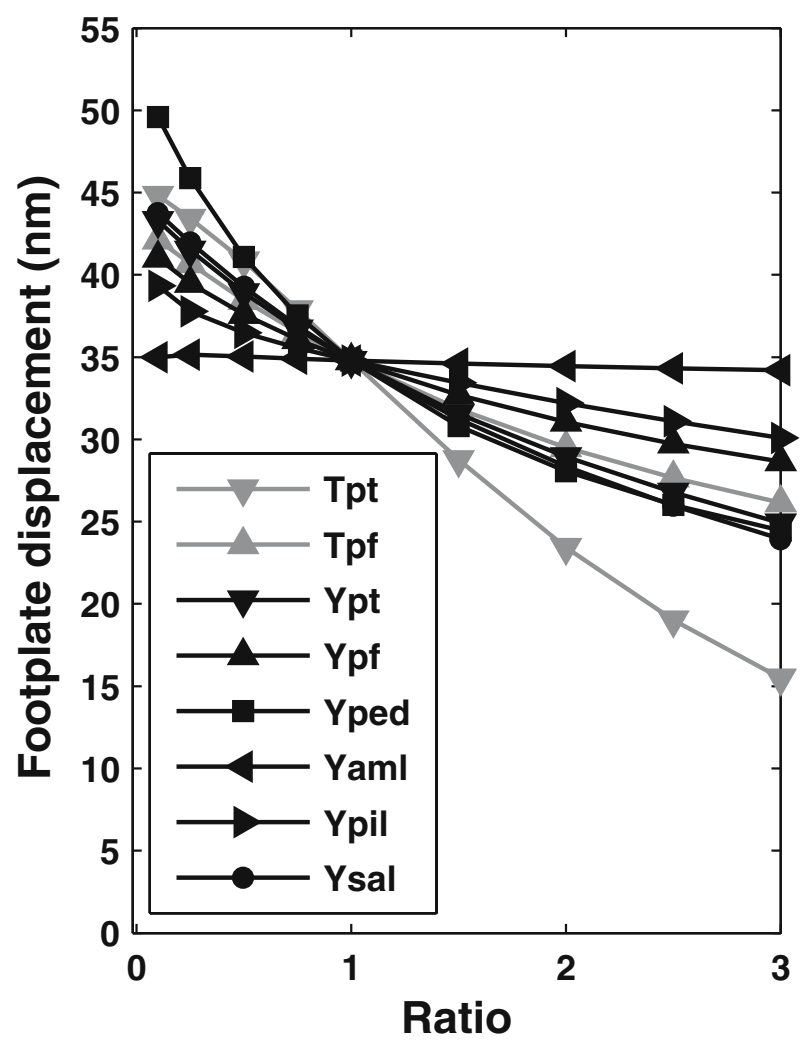

FIG. 6. Effect of each parameter on the footplate displacement. The parameters are (1) the thicknesses of the pars flaccida and pars tensa ( $T_{p f}$ and $T_{p t}$, respectively) and (2) the Young's moduli of the pars flaccida, pars tensa, stapedial annular ligament, anterior mallear ligament, posterior incudal ligament, and pedicle $\left(Y_{p f}, Y_{p t \prime}\right.$ $Y_{\text {sal, }} Y_{\text {aml, }} Y_{\text {pil, }}$ and $Y_{\text {ped, }}$ respectively). 
is much more prominent than that found by Ghosh and Funnell (1995).

\section{Eardrum displacements}

The parameters with the greatest effects on PT displacement are the Young's modulus $\left(Y_{p t}\right)$ and the thickness $\left(T_{p t}\right)$ of the PT itself (Fig. 7). When $T_{p t}$ and $Y_{p t}$ are decreased by a factor of 10 , PT displacements increase by factors of about 37 and 5, respectively. When the same parameters are increased by a factor of 3 , PT displacements decrease by about $67 \%$ and $42 \%$, respectively. The fact that the thickness has a greater effect than the Young's modulus suggests that the bending stiffness of the eardrum is more important than its in-plane, or membrane, stiffness. This is consistent with classical thin-plate theory, which states that the bending stiffness is proportional to the cube of the thickness whereas the inplane stiffness is proportional to the thickness itself.

Four of the remaining six parameters $\left(Y_{p e d}, Y_{p f}, T_{p f}\right.$, and $\left.Y_{\text {sal }}\right)$ have relatively little effect on PT displacements: when they are decreased by a factor of 10 , PT displacements increase by about $23 \%, 12 \%, 15 \%$, and $24 \%$, respectively; when they are increased by a factor of 3, PT displacements decrease by $26 \%, 16 \%, 21 \%$, and $23 \%$, respectively. The influence of the last two

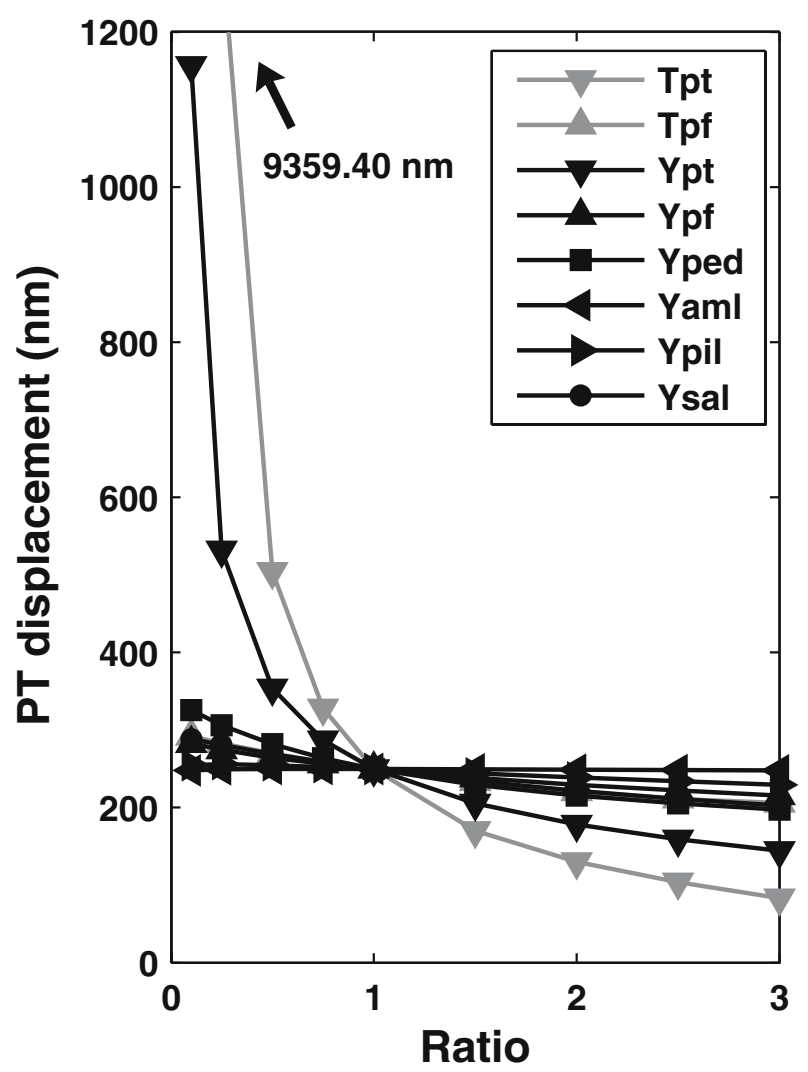

FIG. 7. Effect of each parameter on the pars tensa (PT) displacement.

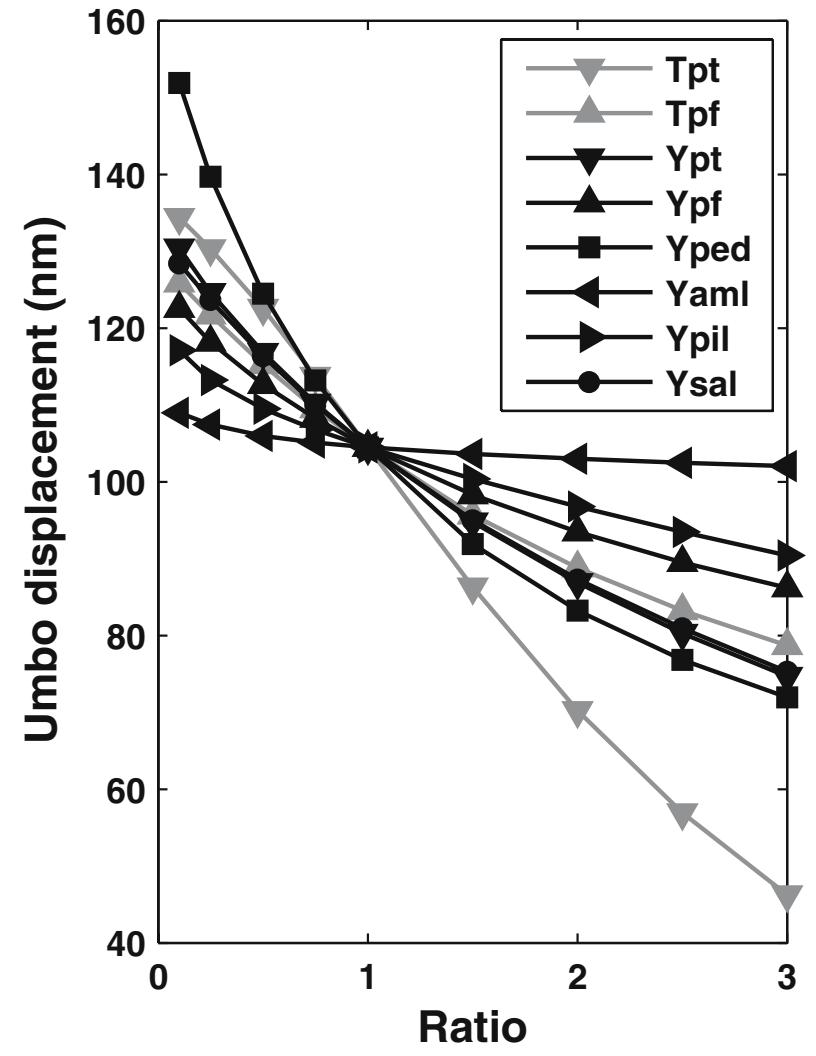

FIG. 8. Effect of each parameter on the umbo displacement.

parameters $\left(Y_{a m l}\right.$ and $\left.Y_{p i l}\right)$ is negligible, with the displacements changing by $2 \%$ or less.

\section{Umbo displacements}

The sensitivity of the umbo to the same eight parameters is shown in Figure 8. There is relatively little difference between the way in which the various parameters influence the umbo and the way in which they influence the footplate. Compared with Figure 6, the most noticeable difference is that $Y_{a m l}$ has little effect on the umbo at low ratios. When $Y_{a m l}$ is decreased by a factor of 10 , umbo displacements increase by only about $4 \%$.

\section{Ossicular lever ratio}

As a measure of the ossicular lever ratio, we calculated the ratio of the umbo displacement to the stapes displacement (U/S ratio) for each parameter (Fig. 9). The vertical scale is expanded because, as noted previously, most of the parameters have approximately the same effects on the umbo and on the footplate.

When the SAL is stiffened, the stapes footplate displacements decrease more than the umbo displacements do, and the $\mathrm{U} / \mathrm{S}$ ratio evidently increases. When the AML becomes less stiff, the malleus is less constrained and lateral motions appear. Lateral motions 


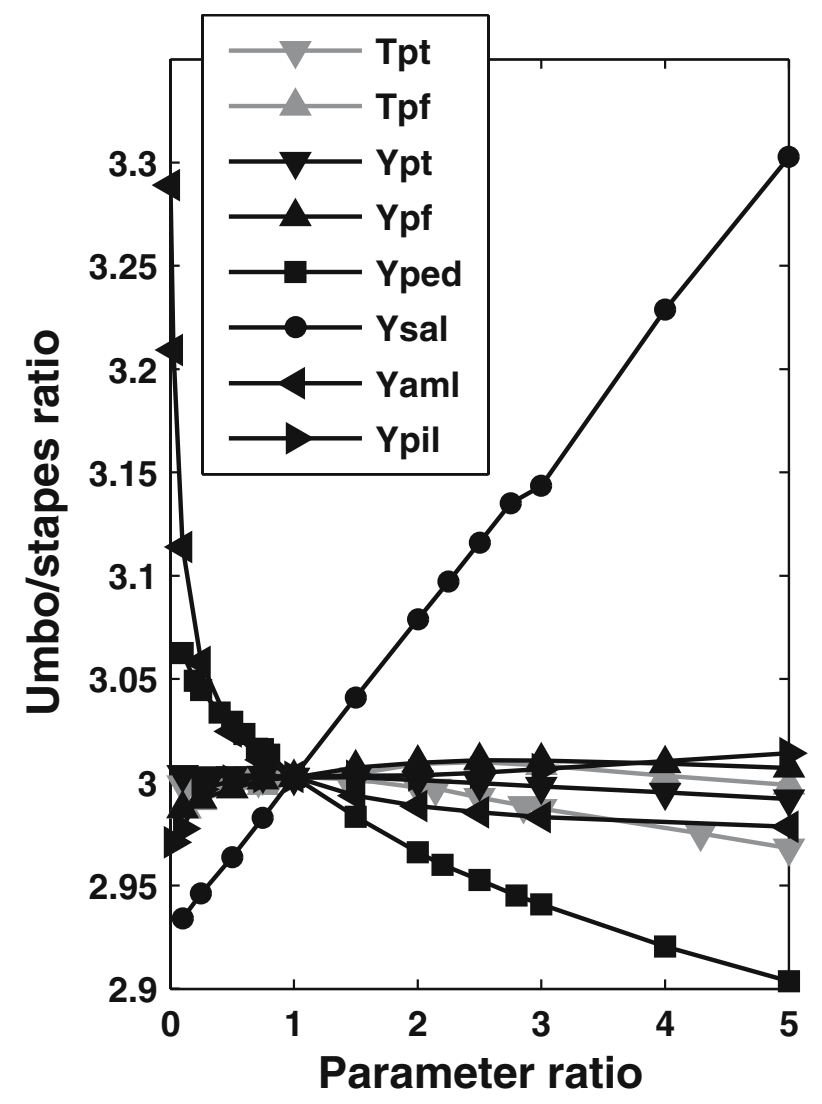

FIG. 9. Effect of each parameter on the umbo/stapes (U/S) ratio, as a measure of the ossicular lever ratio.

are not effective in transferring loads across the ossicles, so stapes footplate displacements decrease and the $\mathrm{U} / \mathrm{S}$ ratio increases.

None of the other parameters had a significant effect on the $\mathrm{U} / \mathrm{S}$ ratio.

\section{Eardrum-manubrium load transfer}

To measure the effectiveness of the load transfer from the eardrum to the ossicles, we calculated the ratio of the PT displacement to the umbo displacement (PT/ $\mathrm{U}$ ratio) for each parameter (Fig. 10), based on the displacement-vector components perpendicular to the plane of the tympanic ring.

When $T_{p t}$ and $Y_{p t}$ are decreased by a factor of 10 , the $\mathrm{PT} / \mathrm{U}$ ratio increases by factors of about 28 and 2 , respectively. When $T_{p t}$ and $Y p t$ are increased by a factor of 5 , the $\mathrm{PT} / \mathrm{U}$ ratio decreases by about $27 \%$ and $23 \%$, respectively. For the other six parameters, the changes in the $\mathrm{PT} / \mathrm{U}$ ratio are smaller and in the opposite direction. As the eardrum becomes less stiff, it cannot displace the ossicles and the $\mathrm{PT} / \mathrm{U}$ ratio increases. On the other hand, as the ossicular system becomes less stiff, the umbo displacements increase and the $\mathrm{PT} / \mathrm{U}$ ratio decreases.
Axis of rotation

In an attempt to understand how the apparent axis of rotation is influenced by the AML and the PIL, we ran simulations on the model for six different cases (see Fig. 11):

- A: The AML and the PIL are kept intact.

- B: The Young's modulus of both the AML and the PIL are set to zero.

- C: Only the Young's modulus of the AML is set to zero.

- D: Only the Young's modulus of the PIL is set to zero.

- E: Only the Young's modulus of the AML is increased by a factor of 10 .

- F: Only the Young's modulus of the PIL is increased by a factor of 10 .

In case $\mathrm{A}$, we can see (as described previously) that the apparent axis of rotation passes through the AML and the PIL.

When both the AML and the PIL have a Young's modulus of zero (case B), the axis of rotation is shifted to the left and completely outside the figure. The parallel contour lines indicate the direction of

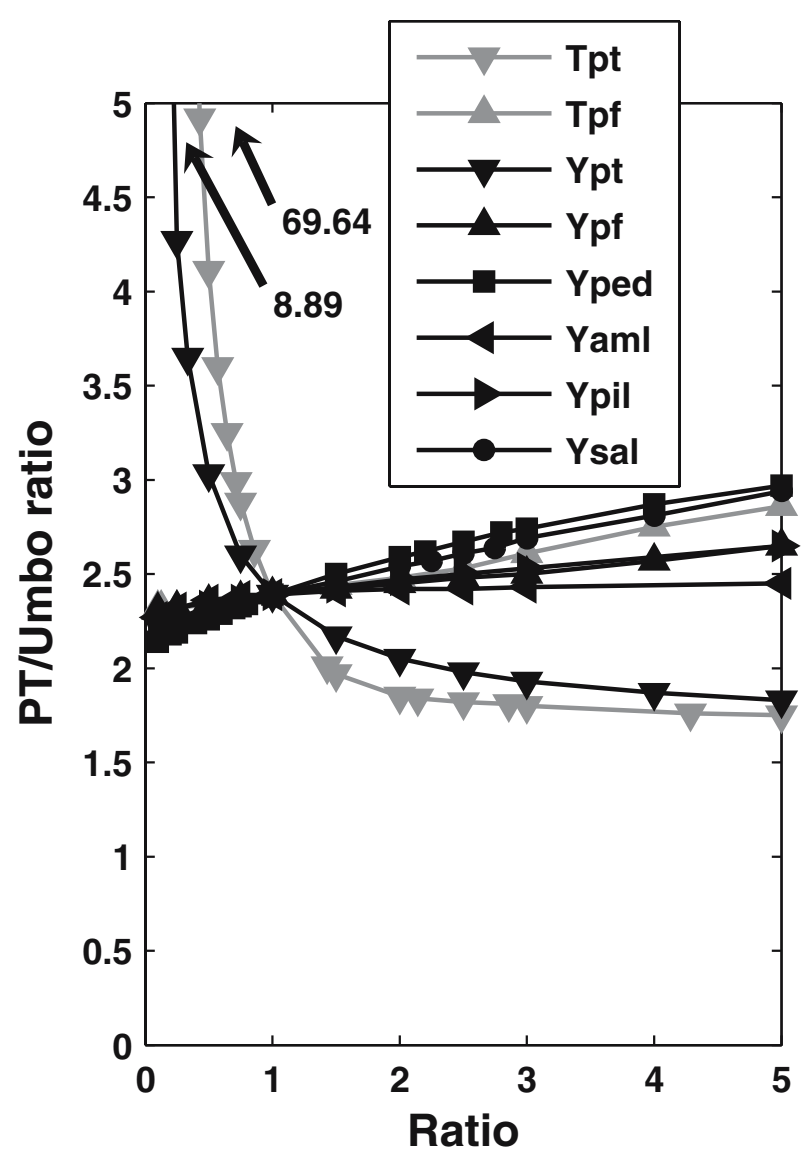

FIG. 10. Effect of each parameter on the PT/umbo (PT/U) ratio, as a measure of the load transfer from the eardrum to the ossicles. 


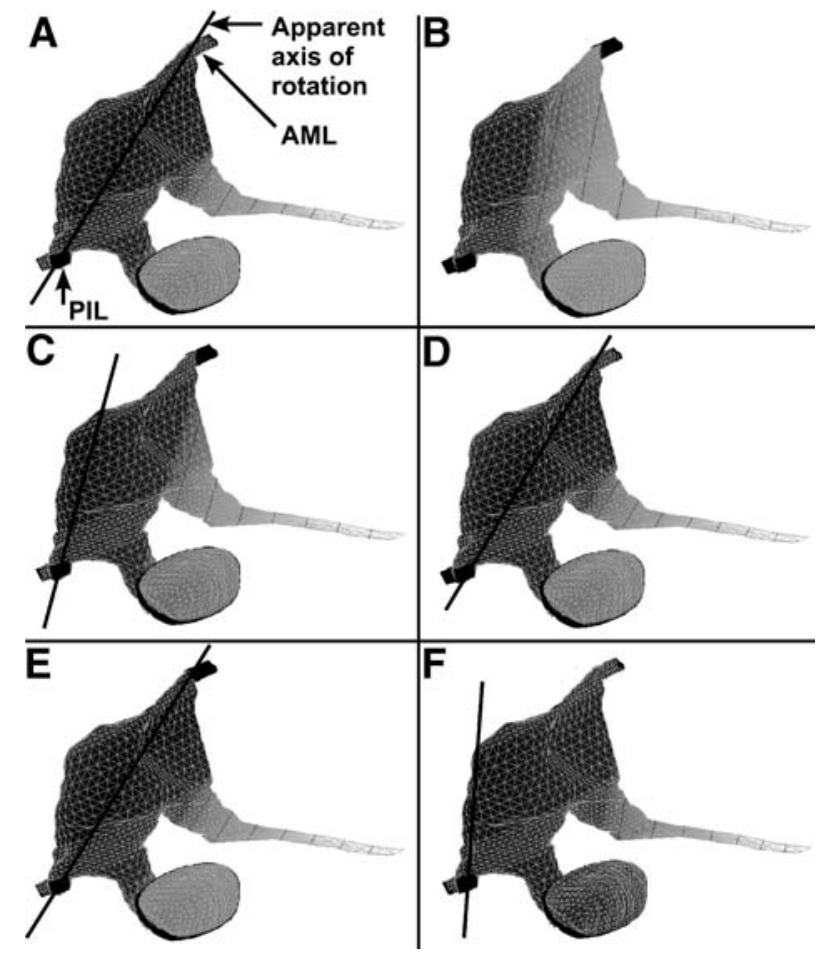

FIG. 11. Effect of removing and stiffening the anterior mallear ligament (AML) and the posterior incudal ligament (PIL) on the apparent axis of rotation. (A) The AML and the PIL are kept intact. (B) The Young's modulus of both the AML and the PIL are set to zero. (C) Only the Young's modulus of the AML is set to zero. (D) only the Young's modulus of the PIL is set to zero. (E) Only the Young's modulus of the AML is increased by a factor of 10. (F) Only the Young's modulus of the PIL is increased by a factor of 10 .

the axis of rotation. Footplate and umbo displacements increase by about $46 \%$ and $29 \%$, respectively. The U/S ratio decreases by about $12 \%$.

When the Young's modulus of the AML is set to zero (case $\mathrm{C}$ ), the anterior end of the axis of rotation is shifted to the left of the AML, and the axis still passes through the PIL. Footplate displacements decrease by only about $3 \%$, and umbo displacements increase by about $7 \%$. The $\mathrm{U} / \mathrm{S}$ ratio is increased by about $10 \%$.

In case D, when the Young's modulus of the PIL is set to zero, the posterior end of the axis of rotation is shifted slightly to the right of the PIL (clearly by a lesser amount than in case $\mathrm{C}$ ), and the axis still passes through the AML. Footplate and umbo displacements both increase by about $18 \%$. The $\mathrm{U} / \mathrm{S}$ ratio is almost unchanged.

When the Young's modulus of the AML is stiffened (case E), the axis of rotation is shifted slightly to the right at both ends. Footplate and umbo displacements both decrease by about $65 \%$. The $\mathrm{U} / \mathrm{S}$ ratio decreases by about $1.5 \%$.

When the PIL is stiffened (case F), the anterior end of the axis of rotation is shifted considerably to the left of the AML. Footplate and umbo displace- ments are decreased to $0.7 \%$ and $1.3 \%$ of their initial values, respectively, and the $\mathrm{U} / \mathrm{S}$ ratio is increased by about a factor of 2 .

In summary,

- When the AML is cut, there is a large axis change but a relatively small change in the ossicular ratio (about 10\%).

- When the PIL is cut, there is a very small axis change and no change in the ossicular ratio.

- When the AML is stiffened, there is practically no change in the axis or in the ossicular ratio.

- When the PIL is stiffened, there is a large axis change but only a moderate change in the ossicular ratio (by a factor of 2 ).

\section{Eardrum shape}

Funnell and Laszlo (1978) developed a finite-element model of the cat eardrum with a rudimentary middle-ear representation. The shape of the eardrum was defined by a normalized radius-of-curvature parameter. They varied the depth of the cone of the eardrum and the degree of curvature, and showed that the shape of the eardrum has a significant influence on middle-ear mechanics. The effects of eardrum shape were also demonstrated in models by Funnell and Decraemer (1996). In this section, we provide preliminary results on the effects of changing the eardrum shape of our model by presenting just one additional eardrum shape. The new shape was obtained by iterative Laplacian smoothing: each node on the eardrum is moved in turn to the position that is the average of the positions of all the nodes to which it is connected. The same process is repeated until none of the nodes moves more than some threshold amount.

Figure 12 shows the eardrum based on the MRM data (A) and the newly shaped eardrum (B), in side and front views, and their displacement patterns. The modified eardrum is steeper near the periphery and near the manubrium, and has a more uniform curvature. The displacement pattern in panel $\mathrm{A}$ is the same as that in Figure 4. In panel B, two areas of maximum displacement are again seen, but they are further away from the manubrium and closer to the annular ring than they are in A, and they are more localized. The maximum displacement in B appears in the posterior region, as it does in A. Maximum eardrum displacement increased from about $250 \mathrm{~nm}$ (A) to about $334 \mathrm{~nm}$ (B). Umbo displacement, however, decreased from about $105 \mathrm{~nm}$ (A) to about $40 \mathrm{~nm}$ (B), and stapes displacement decreased from about $35 \mathrm{~nm}$ (A) to about $13 \mathrm{~nm}$ (B). The ossicular lever ratio ( $\mathrm{U} / \mathrm{S}$ ratio) is thus almost identical in $\mathrm{A}$ and $\mathrm{B}$, but the $\mathrm{PT} / \mathrm{U}$ ratio increased from 2.4 (A) to 


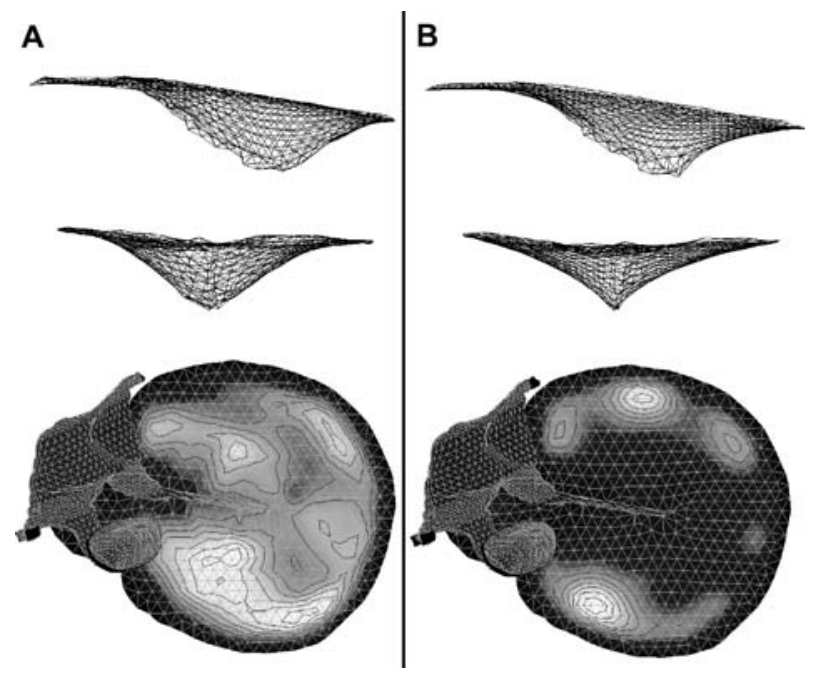

FIG. 12. Effect of changing the shape of the eardrum. (A) eardrum in side and front views, before relaxing the shape. (B) Eardrum in side and front views after relaxing the shape.

8.4 (B), indicating that the modified eardrum shape is less effective in driving the ossicular chain.

\section{COMPARISON WITH EXPERIMENTAL MEASUREMENTS}

To validate our model, we compared its behavior with experimental data. The model has no inertial or damping terms and is therefore applicable to low acoustic frequencies.

\section{Eardrum measurements}

Von Unge et al. (1993) measured the displacement patterns of the gerbil eardrum under quasi-static pressures with a real-time moiré interferometer. Two points of maximum displacement were reported: one in the anterior part, slightly superior to the umbo, and one in the posterior part, somewhat more superiorly. The larger of the two was in the posterior region. These findings are consistent with the results from our eardrum model (Fig. 4) with respect to the positions of the points of maximum displacement. In terms of the displacements themselves, our model values (for low acoustic frequencies) are expected differ from the experimental results because of viscoelastic effects in the quasi-static measurements, and because the experimental pressures were very high and the measured displacements were thus nonlinear.

\section{Ossicular measurements}

Although a number of studies have produced experimental measurements on gerbil middle ears, few have included responses below $1 \mathrm{kHz}$. Ravicz and Rosowski (2004), Overstreet and Ruggero (2002), and Overstreet et al. (2003) dealt exclusively with high-frequency responses. Rosowski et al. (1999), Olson and Cooper (2000), and Ravicz and Rosowski (2004) looked at both low and high frequencies.

Rosowski et al. (1999) and Ravicz and Rosowski (2004) used a probe-tube microphone and a laser Doppler vibrometer to measure stapes displacements at the posterior crus. The laser beam was focused at an angle of between 30 and $45^{\circ}$ relative to the direction of piston-like stapes translation. A cosine correction was used to adjust all laser measurements to a value consistent with the supposed direction of translation. Olson and Cooper (2000) used an interferometer to measure the stapes vibrations at specified points on the footplate.

Only Rosowski et al. (1999) measured displacements at, or at least near, the umbo. To date, no measurements of gerbil eardrum vibrations have been made.

The experimentally measured stapes velocities increase linearly with frequency (i.e., the displacements are constant) up to about $1 \mathrm{kHz}$, indicating that the system is stiffness-dominated over that frequency range. The behavior becomes more complex beyond $1 \mathrm{kHz}$, as inertia and damping become significant. Only the low-frequency results will be used for the comparison with our finite-element model. We averaged the values from Rosowski et al. (1999), Olson and Cooper (2000), and Ravicz and Rosowski (2004) across a frequency range of $100 \mathrm{~Hz}-$ $1 \mathrm{kHz}$. The data from Olson and Cooper (2000) were taken from Ravicz and Rosowski (2004). For comparison with our displacement results, we converted the reported stapes velocities to displacements by dividing by the frequency. These results are compared with our model results in Table 1.

Our umbo displacement differs from that of Rosowski et al. (1999) by about 43\%. Our footplate displacement differs from those of Rosowski et al. (1999) and Olson and Cooper (2000) by approximately $33 \%$ and $27 \%$, respectively, and is almost identical to the value obtained by Ravicz and Rosowski (2004).

The ratio of umbo displacement to stapes footplate displacement $(\mathrm{U} / \mathrm{S})$ gives an indication of the ossicular lever ratio, which is one of the factors often used to define the energy-transfer mechanism of the middle ear. Rosowski et al. (1999) reported an ossicular lever ratio of about 3.5. In our model, the ratio is 3.0. Keeping in mind the variability of the experimental data, this agrees quite well with their value. The value of 3.5 is outside the range of values seen in Figure 9 for different model parameters. As noted above, none of the parameters have a strong 
TABLE 1

\begin{tabular}{ccccc}
\hline & \multicolumn{4}{c}{ Summary of gerbil middle-ear experiments } \\
\hline Displacements $(\mathrm{nm})$ & Model & Rosowski et al. (1999) & Olson and Cooper (2000) & Ravicz and Rosowski (2004) \\
\hline Pars tensa & 250 & - & - & - \\
Umbo & 104.5 & 183.8 & - & - \\
Footplate & 34.8 & 52.5 & 47.7 & 35 \\
\hline
\end{tabular}

effect on $\mathrm{U} / \mathrm{S}$, but varying the geometry of the ossicles and ligaments presumably would.

\section{Ligament measurements}

The apparent axis of rotation observed in our model is similar to the classical description given in the literature for low frequencies, namely, passing from the AML to the PIL.

Nakajima et al. (2004) determined the effects both of stiffening and of removing the AML and the PIL in fresh human ossicles. Sound-induced umbo and stapes velocities were measured by laser vibrometry. Stiffening of the AML resulted in a decrease in umbo and stapes displacements by a factor of 2 or less. In our model, the umbo and stapes displacements decreased by $65 \%$. No experimental studies have included the effects of stiffening the PIL.

Experimental removal of the AML resulted in an increase in umbo displacements of less than $60 \%$ and a change in footplate displacements of less than $30 \%$. Removal of the PIL resulted in an increase in umbo displacements of less than $40 \%$ and a change in footplate displacements of less than $15 \%$. In our model, when the stiffness of the AML was set to zero, the umbo displacements increased by only $7 \%$ and the stapes footplate displacements decreased by only $3 \%$. When the stiffness of the PIL was set to zero, the umbo and stapes displacements both increased by $18 \%$. If the middle-ear muscles were added to the model, these changes would presumably be smaller.

Nakajima et al. (2004) concluded that removal of either the AML or the PIL affects ossicular motion only minimally at low frequencies. Large and complex changes were reported, however, when both the AML and the PIL were removed. These results are qualitatively consistent with our findings.

\section{DISCUSSION}

A finite-element model of the gerbil middle ear was developed. The behavior of this model in response to a low-frequency sound pressure of $1 \mathrm{~Pa}$ was analyzed. Sensitivity tests were performed to evaluate the significance of the different parameters in the finite-element model. To validate the model, we compared our results with experimental data from the literature.

The Young's modulus and the thickness of the PT had the greatest effect on PT displacements and, along with the Young's moduli of the pedicle and the SAL, on umbo and stapes displacements. The Young's moduli of the PIL and the AML had the least effect on the model. The ossicular lever ratio (umbo/stapes ratio) was relatively insensitive to all parameters. The coupling between the eardrum and the manubrium (PT/umbo ratio) was found to be highly affected by the PT parameters, and to a lesser extent by the thickness of the PF and by the Young's moduli of the pedicle and the SAL. Finally, the shape of the eardrum was shown to have an effect on the displacements of the middle-ear components, and on the displacement profile of the eardrum itself.

Overall, our finite-element model demonstrates good agreement with experimental data. The displacement pattern of the PT in our model is similar in shape to profiles measured experimentally (Von Unge et al. 1993). The umbo and stapes displacements, as well as the ossicular displacement ratio, compare satisfactorily with low-frequency measurements from Rosowski et al. (1999), Olson and Cooper (2000), and Ravicz and Rosowski (2004). The ossicular axis of rotation extended from the AML to the PIL, corresponding to the classical description, and the motion of the stapes is piston-like, in agreement with the lowfrequency observations of Decraemer et al. (2005).

The axis of rotation was influenced by the AML when it was cut, by the PIL when it was stiffened, and when they were both cut. The corresponding changes in ossicular displacements are comparable to those of Nakajima et al. (2004).

The umbo and stapes displacements decreased by $65 \%$ when the Young's modulus of the AML was increased by a factor of 10 . This is comparable to the findings in human models by Abou-Khalil et al. (2001) and Huber et al. (2003).

The present model is a step toward detailed quantitative models that can be validated against the considerable body of experimental data on the gerbil middle ear, and in turn can help explain those data.

Several enhancements should be undertaken to improve the model. (1) Inertial and damping properties should be included in the model to permit 
simulations at higher frequencies. This would allow comparison with the nonpiston-like stapes motion observed by Decraemer et al. (2005). (2) The study should be extended to nonlinear analysis. The large (nonlinear) displacements could be compared with recent moiré data from Decraemer's laboratory (Decraemer et al. 2003; Dirckx and Decraemer 2003). (3) In our study, the periphery of the eardrum was assumed to be fixed. If an explicit representation of the fibrocartilaginous ring (including the smooth muscle cells) were included in the model, the behavior of the model could be compared with the results of studies in which the physiological effects of the smooth muscle were investigated through the administration of drugs known to relax or contract smooth muscle (Yang and Henson 2002). (4) The study of the effects of different eardrum shapes should be extended. (5) A more thorough representation of the pedicle, the incudomallear and incudostapedial joints, the stapedius and tensor tympani muscles, and the ligament/bone connections would serve to improve our understanding of ossicular motion. One specific area to be clarified is whether the connection of the anterior process of the malleus to the wall of the middle-ear air cavity is bony, as observed by Rosowski et al. (1999), or ligamentous as suggested by our histological and MRM data. (6) Orthotropic material properties for the eardrum should be investigated and the resulting behavior compared with the isotropic case.

Displacement measurements on the gerbil eardrum need to be made to validate this model properly, and measurements of displacement vectors at multiple locations on the ossicles would also be helpful.

\section{ACKNOWLEDGMENTS}

This work was supported by the Canadian Institutes of Health Research and the Natural Sciences and Engineering Research Council (Canada). We thank M.M. Henson and O.W. Henson Jr., (UNC-Chapel Hill) and the Center for In Vivo Microscopy (Duke University) for the data used for the MRM-based model. We also thank W.F. Decraemer (University of Antwerp, Belgium) for the x-ray micro-CT and moiré data, and M. von Unge (Karolinska Institute, Sweden) for the histological serial sections. Finally, we thank J. Lauzière for editing the manuscript.

\section{REFERENCES}

Abou-Khalil S, Funnell WRJ, Zeitouni AG, Schloss MD, Rappaport J (2001) Finite-element modeling of anterior mallear ligament fixation. Toronto, Eastern Section Meeting, Triological Soc.
Beer HJ, Bornitz M, Hardtke HJ, Schmidt R, Hofmann G, Vogel U, Zahnert T, HÜttenbrink KB. Modelling of components of the human middle ear and simulation of their dynamic behavior. Audiol. Neuro-otol. 4:156-162, 1999.

Bornitz M, Zahnert T, HardtKe HJ, HÜtTEnbrink KB. Identification of parameters for the middle ear model. Audiol. Neuro-otol. 4:163-169, 1999.

Decraemer WF, Khanna SM. Three dimensional vibration of the ossicular chain in the cat. Vibration measurements by laser techniques: advances and applications, Proc. SPIE 4072: 401-411, 2000.

Decraemer WF, Gea SLR, Dirckx JJJ (2003) Three-dimensional displacement of the gerbil ossicular chain under static pressure changes. 26th ARO MidWinter Mtg.

Decraemer WF, Khanna SM, de la Rochefoucauld O, Dong W, Olson ES (2005) Is the scala vestibuli pressure influenced by non-piston like stapes motion components? An experimental approach. Auditory mechanisms: processes and models. Portland (OR), Proc. Ninth International Mechanics of Hearing Workshop, 119-120.

Dirckx JJJ, Decraemer WF. Phase shift moiré apparatus for automatic 3-D surface measurement. Rev. Sci. Instrum. 60: 3698-3701, 1989.

Dirckx JJJ, Decraemer WF. Automatic calibration method for phase shift shadow moiré interferometry. Appl. Opt. 29:1474-1476, 1990.

Dirckx JJJ, Decraemer WF. Effect of middle ear components on eardrum quasi-static deformation. Hear. Res. 157:124-137, 2001.

Dirckx JJJ, Decraemer WF (2003) Pressure induced eardrum deformation at progressive stages of middle ear dissection. 26th ARO MidWinter Mtg.

Evans FG (1973) Mechanical Properties of Bone. Springfield, IL, Charles Thomas, p. 35.

Fay J, Puria S, Decraemer WF, Steele C. Three approaches for estimating the elastic modulus of the tympanic membrane. J. Biomech. 38:1807-1815, 2005.

FunNell WRJ, Laszlo CA. Modeling of the cat eardrum as a thin shell using the finite-element method. J. Acoust. Soc. Am. 63:1461-1467, 1978.

FunNell WRJ, Decraemer WF (1996) Finite-element modelling of the cat middle ear with elastically suspended malleus and incus. 19th ARO MidWinter Meeting.

Funnell WRJ, Decraemer WF, Khanna SM. On the damped frequency response of a finite-element model of the cat eardrum. J. Acoust. Soc. Am. 81:1851-1859, 1987.

Funnell WRJ, Decraemer WF, von Unge M, Dirckx JJJ (1999) Finiteelement modelling of the gerbil eardrum and middle ear. 22nd ARO MidWinter Meeting.

Funnell WrJ, Decraemer WF, von Unge M, Dirckx JJJ (2000) Finiteelement modelling of the gerbil eardrum and middle ear. 23rd ARO MidWinter Meeting.

Funnell WRJ, Siah TH, McKee MD, Daniel SJ, Decraemer WF. On the coupling between the incus and the stapes in the cat. J. Acoust. Res. Otol. 6:9-18, 2005.

GHosh SS, FunNell WRJ (1995) On the effects of incudostapedial joint flexibility in a finite-element model of the cat middle ear. Proc. IEEE-EMBS 17th Annual Conference and 21st Can. Med. \& Biol. Eng. Conf., Montreal, 1437-1438.

Guinan JJ JR., Peake WT. Middle-ear characteristics of anesthetized cats. J. Acoust. Soc. Am. 41:1237-1261, 1967.

GuNDERSEN T. Holographic vibration analysis of the ossicular chain. Acta Otolaryngol. 82:16-25, 1976.

Huber A, Koike T, Wada H, Nandapalan V, Fisch U. Fixation of the anterior mallear ligament: diagnosis and consequences for hearing results in stapes surgery. Ann. Otol. Rhinol. Laryngol. 112:348-355, 2003 
KoIke T, WADA H, Kobayashi T. Modeling of the human middle ear using the finite-element method. J. Acoust. Soc. Am. 111:13061317, 2002.

Kuypers LC, Dirckx JJJ, Decraemer WF, Timmermans J-P. Thickness of the gerbil tympanic membrane measured with confocal microscopy. Hear. Res. 209:42-52, 2005.

LADAK HM, FunNell WRJ. Finite-element modeling of the normal and surgically repaired cat middle ear. J. Acoust. Soc. Am. 100: 933-944, 1996.

Lynch TJ, Nedzelnitsky V, Peake WT. Input impedance of the cochlea in cat. J. Acoust. Soc. Am. 72:108-130, 1982.

Mente PL, Lewis JL. Elastic modulus of calcified cartilage is an order of magnitude less than that of subchondral bone. J. Orthop. Res. 12:637-647, 1994.

Nakajima HH, Peake WT, Rosowski JJ, Merchant SN (2004) Ossicular ligaments and the axis of rotation. 27th ARO MidWinter Meeting.

OAKs E (1967) Structure and function of inflated middle ears of rodents. Ph.D. thesis, Yale University, New Haven.

Olson E, CoOper N (2000) Stapes motion and scala vestibuli pressure in gerbil. 23rd ARO MidWinter Meeting.

Overstreet EH, Ruggero MA. Development of wideband middleear transmission in the Mongolian gerbil. J. Acoust. Soc. Am. 111:261-270, 2002.

Overstreet EH, Richter CP, Temchin AN, Cheatham MA, Ruggero MA. High-frequency sensitivity of the mature gerbil cochlea and its development. Audiol. Neuro-otol. 8:19-27, 2003.

Prendergast PJ, Ferris P, Rice HJ, Blayney AW. Vibroacoustic modelling of the outer and middle ear using the finite-element method. Audiol. Neuro-otol. 4:185-191, 1999.

QI L, Mikhael CS, Funnell WRJ. Application of the Taguchi method to sensitivity analysis of a middle-ear finite-element model. Proc. 28th Ann. Conf. Can. Med. Biol. Eng. Soc., 153-156, 2004

Ravicz ME, Rosowski JJ (2004) High-frequency sound transmission through the gerbil middle ear. 27th ARO MidWinter Meeting.

Robles L, Temchin A, Fan Y-H, Cai H, Ruggero M (2006) Vibrations of the stapes and the long and lenticular processes of the incus in the chinchilla middle ear. 29th ARO MidWinter Meeting.

Rosowski JJ, Ravicz ME, Teoh SW, Flandermeyer D. Measurements of middle-ear function in the Mongolian gerbil, a specialized mammalian ear. Audiol. Neuro-Otol. 4(3-4):129-136, 1999.

Speirs AD, Hotz MA, Oxland TR, Hausler R, Nolte L-P. Biomechanical properties of sterilized human auditory ossicles. J. Biomech. 32:485-491, 1999.

Sun Q, Gan RZ, Chang KH, Dormer KJ. Computer-integrated finiteelement modeling of human middle ear. Biomech. Model. Mechanobiol. 1:109-122, 2002.

Van Wijhe RG, Funnell WRJ, Henson OW Jr., Henson MM. Development of a finite-element model of the middle ear of the moustached bat. Proc. 26th Ann. Conf. Can. Med. Biol. Eng. Soc., 20-21, 2000.

Von Unge M, Decraemer WF, Bagger-SJöbäck D, Dirckx JJJ. Displacement of the gerbil tympanic membrane under static pressure variations measured with a real-time differential moiré interferometer. Hear. Res. 70:229-242, 1993.

Wada H, Metoki T, Kobayashi T. Analysis of dynamic behavior of human middle ear using a finite-element method. J. Acoust. Soc. Am. 92:3157-3168, 1992.

YANG X, Henson OW JR. Smooth muscle in the annulus fibrosus of the tympanic membrane: physiological effects on sound transmission in the gerbil. Hear. Res. 164:105-114, 2002. 